\title{
Late bronchus perforation due to spinal implant compression
}

\begin{abstract}
We report a rare case of perforation of the intermediate bronchus by a loosened osteosynthetic implant, 8 years after the patient had undergone surgery for right thoracic scoliosis with the VDS instrumentation. Due to chronic lung inflammatory alteration caused by bronchial obstruction, lower bi-lobectomy was performed. We believe that spinal growth and progression of idiopathic scoliosis can lead to implant failure, while the variance in intrathoracic topographic relations and respiratory excursion facilitate it's penetration into the bronchus. In order to impede foreign body perforation of mediastinal structures and to reduce its complications, preventive methods, along with timely diagnostics, are required. Atelectatic and chronic inflammatory alterations that compromise adequate lung ventilation could require a resection procedure.
\end{abstract}

Keywords: Foreign body; Lobectomy; Thoracic scoliosis; VDS instrumentation
Volume 6 Issue 5 - 2016

\author{
Irena Sokolović,' Zoran Slobodnjak, ${ }^{2}$ Ivica \\ Mažuranić ${ }^{3}$ \\ 'Department of Anesthesiology, Reanimatology and Intensive \\ Care, Clinical Center for Pulmonary Diseases Jordanovac, \\ University Hospital Centre Zagreb, Croatia \\ ${ }^{2}$ Department of Thoracic Surgery Jordanovac, Clinical Center \\ for Pulmonary Diseases Jordanovac, University Hospital Centre \\ Zagreb, Croatia \\ ${ }^{3}$ Department of Radiology Jordanovac, Clinical Center for \\ Pulmonary Diseases Jordanovac, University Hospital Centre \\ Zagreb, Croatia
}
Correspondence: Irena Sokolovic, Department of Anesthesiology, Reanimatology and Intensive Care, Clinical Center for Pulmonary Diseases Jordanovac, University Hospital Centre Zagreb, Jordanovac 104, 10000 Zagreb, Croatia, Tel +385 I2385 I I2, Email isokolovic2@gmail.com

Received: November 19, 2016 | Published: December 28, 2016
Abbreviations: VDS, Ventral derotationspondylodesis; VC, vital capacity; FEV1, forced expiratory volume in 1 second; TI, tiffeneau index; DLCO, diffusing capacity; $\mathrm{CT}$, computed tomography

\section{Introduction}

Surgical treatment of scoliosis has a varying but high rate of complications, such as: lethal outcome in less than $1 \%$, neurological damage, irreversible loss of the normal active range of the movement in the vertebral column, strain on un-fused vertebrae, post-surgery pain $(19 \%$ of patients required re-operation within 2 to 8 years after surgery), deep wound infections (5-10\%), bone deterioration due to inflammatory response to metallic implant, curvature progression due to failure of instrumentation, decompensation and increased sagittal deformity ("flat back"), torso deformity ("flail chest"). ${ }^{1}$ A choice of treatment for thoracic scoliosis is anterior instrumentation surgery because better correction can be obtained with shorter fusion levels. ${ }^{2}$ However, anterior instrumentation carries a greater risk for penetration of vital structures by sprung fragment of inner vertebral fixator, as well as the effect of disrupted chest cage on pulmonary function.

Spinal fusion surgery is recommended when the magnitude of curvature exceeds $45^{\circ}$, but it is expected for $1 / 3$ of patients to lose all postoperative correction within 1-10 years post-surgery. ${ }^{3}$ It has been noted that $1.5 \%$ of the screws inserted in the thoracic level were displaced, but they did not cause any adversely effect on longterm results. ${ }^{1}$ We present a rare case of erosion of the intermediate bronchus caused by dislocated osteosynthetic material implanted for the treatment of thoracic scoliosis in 1995. To our knowledge, only one case of erosion of the bronchus after surgical treatment of scoliosis has been reported. ${ }^{4}$ Both of patients presented with long-time history of cough, haemoptysis and dyspnoea.

\section{Case presentation}

In June of 1995 a 17-year-old patient underwent spinal instrumentation and fusion through placement of VDS implants for the treatment of idiopathic right thoracic scoliosis. The type of curve by Lenke classification system for scoliosis was 2AN (curve length from the 5 th thoracic through the 1 st lumbar vertebra), with measured Cobb's angle of $90^{\circ}$. The operation was performed through a right thoracotomy. The VDS implants were inserted laterally on the convex side of the vertebral bodies and correction was done by compression and derotation. In order to achieve a more stable and stronger fixation, Luque's rod was inserted. The patient has received clinical and radiographic three-year follow-up. In 2003 the patient was admitted to our surgical clinic because of her persistent cough, sub-febrility, dyspnoea and haemoptysis. Spirometry results showed a mid-grade obstructive-restrictive disorder ( $\downarrow$ VC: $2.46 ; \downarrow$ FEV1: 1.60 ; $\downarrow$ TI: $65 \%$; $\downarrow$ DLCO: 63\%). Radiographic analysis disclosed a loosened fragment of an inner vertebral fixator at the level of 7 th and 8 th thoracic vertebra with a protrusion into the intermediate bronchus. Fibro-bronchoscopy has confirmed a perforation of the intermediate bronchus caused by a metallic foreign body. CT scan revealed the fractured osteosynthetic material at the level of 7th and 8th thoracic vertebra and its endobronchial migration with a significant pleural encapsulation that compressed the right lower lobe parenchyma. Due to distinct granulations with proximal and distal expanding, the intermediate bronchus was substantially stenosed with subsequent atelectatic changes of the lower lobe parenchyma that required surgical treatment.

After induction of general anaesthesia, patient was intubated with a left-sided double-lumen tracheal tube and pressure-controlled one-lung ventilation was provided by a closed-circuit anaesthesia ventilator. The ventilation volume was reduced to $6 \mathrm{ml} / \mathrm{kg}$ with the pressure in the airways less than $30 \mathrm{cmH} 2 \mathrm{O}$, as well as an increased concentration of oxygen in inspiratory admixture and positive endexpiratory pressure. A perioperative thoracic epidural analgesia was provided with the catheter inserted between 4th and 5th thoracic vertebra. Anaesthesia was maintained with 1 minimum alveolar 
concentration of volatile anaesthetic and neuromuscular blocking drugs administered. The patient was placed in the lateral decubitus position and right thoracotomy was performed. Following the decortication of the lung and exposure of vertebral bodies, an inner vertebral fixator was entirely removed. Due to inadequate ventilation, lower bi-lobectomy was performed. Pathohistological analysis has proven chronic inflammatory alteration of resected lung lobes. Close clinical and radiographic follow-up data till 2012 presented full recovery.

\section{Discussion}

Migration of foreign bodies from an extra-pulmonary site to the endobronchial space is uncommon, but feasible cause of bronchial obstruction. Numerous cases of foreign-body migration after mediastinoscopy, cardiothoracic and orthopaedic procedures, as well as its features and facilitating factors, have been debated. ${ }^{5-9} \mathrm{~A}$ ubiquitous orthopaedic procedure - correction of scoliosis with VDS implant alterates topographic and dynamic relations of thoracic vertebrae to adjacent structures and between themselves. A stiffer implant achieves a better correction, but causes higher axial and transverse screw forces. Axial tensile forces act on the screws fixed to the cranial vertebra and the middle vertebra, while axial compressive forces act on the other screws. The transverse force may weaken the bone around the thread and lower the pull-out resistance against the axial force. A strong correction at the cranial segment leads to high axial and transverse screw forces in the furthest cranial screw and thus to high risk of screw pull-out. ${ }^{10}$ The cranial end vertebra is also the vertebra where most screw pull-outs and subsequent lesions of adjacent structures have been observed.

We believe that alteration of dynamic relations, due to amplification of axial and transverse screw forces caused by spinal growth and curvature progression, as well as metal fatigue caused by repetitive stress, has induced the dislocation of osteosynthetic implant. Continual stress on an implant may result in the breakdown of the construction, while the respiratory excursion can lead to the perforation of adjacent structures by a migrated fragments. Bone density influences biomechanical properties that determine the axial pull-out strength of anterior spinal implants. In order to facilitate the preoperative planning and reduce initial implant dislocation to a minimum, absorptiometric determination of bone quality (QCT, DEXA, HRCT) at the operation site before surgery should be obtained. ${ }^{11}$ Bone maturation can also be specified by Risser sign (ossification of the posterior part of the iliac apophysis quantified at six grades: $0-$ no apophysis visible; $1-25 \%$ excursion; $2-50 \%$ excursion; $3-75 \%$ excursion; 4 - complete excursion; 5 - fusion of the apophysis to the ilium), which is negatively correlated with curvature progression. ${ }^{12}$

Distinctive feature of thoracic scoliosis is an amended spinal posture and topographic relation to the tracheobronchial tree, as well as to the other adjacent structures. Preoperative evaluation of the spatial relations between vertebral bodies and mediastinal structures and theirs relative migration due to the anterior correction and instrumentation in right thoracic scoliosis is an essential element of patient's preparation and follow-up. A case of mechanical erosion of the descending thoracic aorta caused by a loose screw on an inner vertebral fixator implanted for the treatment of scoliosis, has been reported. ${ }^{13}$ It has been noted that the thoracic aorta in scoliosis patients is positioned more posterior-lateral, but because of the correction migrates in a more anterior-medial position. ${ }^{14}$ We assume that the analogic alteration in spatial relations also occurs with the tracheobronchial tree. Due to those changeovers, it is necessary to do adequate radiographic estimation of patients with a thorax "at risk", in order to single out the proper moment and procedure.

Although preoperative and intraoperative preventive methods greatly decrease the presumption of tracheobronchial lesion by a dislocated osteosynthetic implant, erosion and perforation of the bronchus still remain a foreseen complication of spinal instrumentation in thoracic scoliosis. Bronchial perforation is uncommon, but has a mortality of nearly $30 \% .{ }^{15}$ Most frequently, a patient presents with dyspnoea and haemoptysis, which was also the case of our patient. Voluminous pneumothorax with continued air leak occurs when the rupture site is in direct communication with the pleural cavity (bronchopleural fistula). ${ }^{15}$. Subsequent atelectasis due to bronchial stenosis is most often evolved before the diagnosis is established. A computed tomography scan and fiberoptic bronchoscopy provide definitive diagnosis. With chronic atelectasis, secondary infection complicates the process inducing self-perpetuating chronic inflammatory involvement of the parenchyma resulting in bronchiectasis and destroyed lobe. ${ }^{16}$ When recurrent infection results in irreversible damage of the lobe, lobectomy is considered safe and effective therapy of choice. ${ }^{17}$ (Figures 1-4).

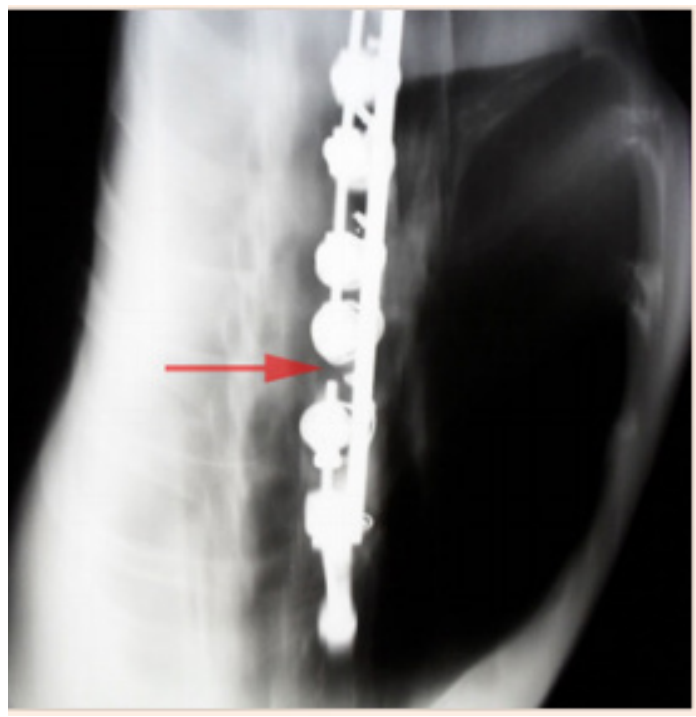

Figure I Tomogram: the fracture of an osteosynthetic implant at the level of $7^{\text {th }}$ and $8^{\text {th }}$ thoracic vertebra.

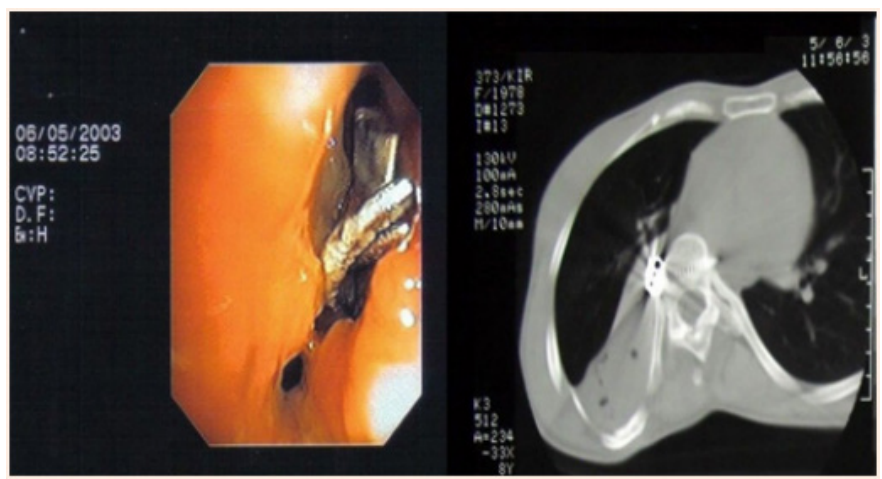

Figure 2 Fibro-bronchoscopy: the perforation of the intermediate bronchus caused by a metallic foreign body. Distinct granulations with proximal and distal expanding. CT: a loosened fragment of an inner vertebral fixator at the level of 7 th and 8 th thoracic vertebra with a protrusion into the intermediate bronchus. 


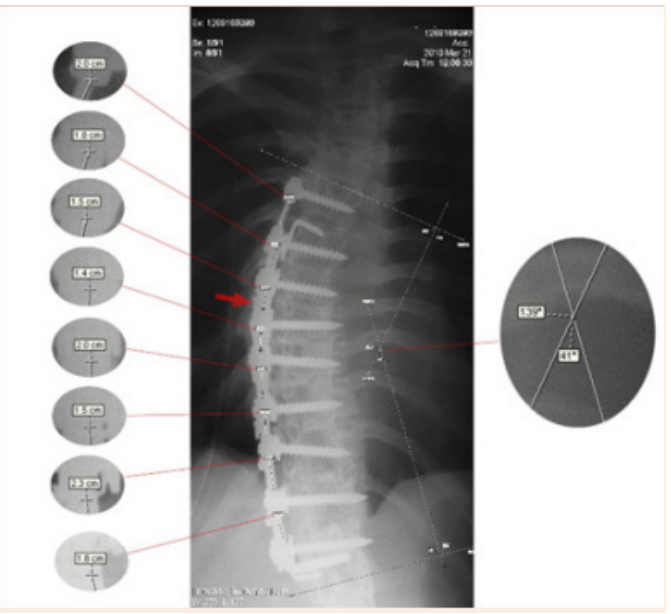

Figure 3 1995: the sum of the distance between implanted screws: 14,3cm; Cobb's angle: $41^{\circ}$.

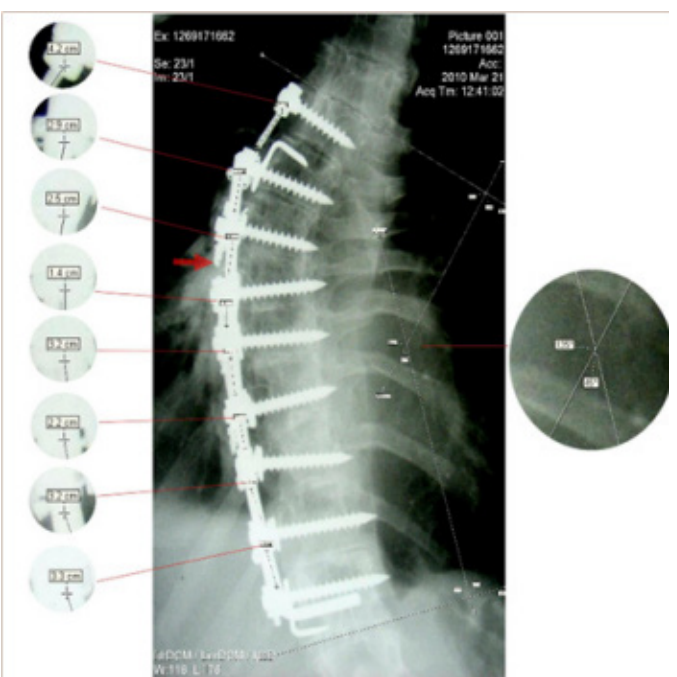

Figure 4 2003: the sum of the distance between implanted screws: $22,9 \mathrm{~cm}$; Cobb's angle: $45^{\circ}$

\section{Conclusion}

Bronchial perforation caused by a loosened osteosynthetic implant is rare, but potential and severe complication of spinal instrumentation in thoracic scoliosis. We reported a rare case of perforation of the intermediate bronchus by a dislocated osteosynthetic implant with subsequent atelectatic and chronic inflammatory alteration of lung parenchyma that required lower bi-lobectomy. The risk for that complication can be determined by preoperative evaluation of spatial relations between vertebral bodies and mediastinal structures and theirs relative migration due to anterior correction and instrumentation. In order to estimate the concerned risk, we have suggested diagnostic procedures and radiographic criteria. Timely diagnosis of bronchial perforation is of great importance, because of lung destruction due to bronchial obstruction or stenosis. In order to put an end to doubt whether the presumptive cause of sypmtoms, such as dyspnoea and haemoptysis, in a patient who underwent spinal instrumentation, is hidden behind the bronchial perforation caused by a loosened osteosynthetic implant, CT and fibro-bronchoscopy need to be done. If the lung ventilation is inadequate, a resection procedure could be required. Lobectomy may be necessary when recurrent infection results in irreversible damage of the lobe.

\section{Acknowledgements}

The authors thank Ante Zovko, radiologic technologist (Department of Radiology at the Clinic for Pulmonary Diseases Jordanovac), for his assistance in radiographic processing.

\section{Conflict of interest}

The authors whose names are listed immediately below certify that they have NO affiliations with or involvement in any organization or entity with any financial interest or non-financial interest in the subject matter or materials discussed in this manuscript.

\section{Funding}

None.

\section{References}

1. Weiss HR, Goodall D. Rate of complications in scoliosis surgery - a systematic review of the Pub Med literature. Scoliosis. 2008;3:9.

2. Maruyama T, Takeshita K. Surgical treatment of scoliosis: a review of techniques currently applied. Scoliosis. 2008;3:6.

3. Weiss HR, Bess S, Wong MS, et al. Adolescent idiopathic scoliiosis - to operate or not? A debate article. Patient Saf Surg. 2008;2(1):25.

4. Wong K, Waters C M, Walesba RK. Haemoptysis after Dwyer instrumentation for idiopathic scoliosis. J R Soc Med. 1991;84(11):685686.

5. Öztuna F, Bülbül Y, Özlü T. An unusual endobronchial foreign body: a gauze that migrated from the mediastinum. Respiration. 2005;72(5):543-545.

6. Dasgupta A, Mehta AC, Wiedemann HP, et al. Erosion of implantable cardioverter defibrilator patch electrode into airways. Chest. 1998;113(1):252-254.

7. Foster GT, Chetty KG, Mahutte K, et al. Haemoptysis due to migration of a fractured Kirschner wire. Chest. 2001;119(4):1285-1286.

8. Nakayama M, Gika M, Fukuda H, et al. Migration of a Kirschner wire from the clavicle into the intrathoracic trachea. Ann Thorac Surg. 2009;88(2):653-654.

9. Lyons FA, Rockwood CA. Migration of pins in operations on the shoulder. J Bone Joint Surg An. 1990;72(8):1262-1267.

10. Rohlmann A, Richter M, Zander T, et al. Effect of different surgical strategies on screw forces after correction of scoliosis with VDS implant. Eur Spine J. 2006;15(4):457-464.

11. Eysel P, Schwitalle M, Oberstein A, et al. Preoperative estimation of screw fixation strength in vertebral bodies. Spine. 1998;23(2):174-180.

12. Kotwicki T. Improved accuracy in Risser sign grading with lateral spinal radiography. Eur Spine J. 2008;17(12):1676-1685.

13. Sokolić J, Šoša T, Ugljen R, et al. Extrinsic erosion of the descending aorta by a vertebral fixator. Tex Heart Inst J. 1991;18(2):136-139.

14. Bullmann V, Fallenberg EM, Meier N, et al. The position of the aorta relative to the spine before and after anterior instrumentation in right thoracic scoliosis. Spine. 2006;31(15):1706-1713.

15. Harvey-Smith W, Bush W, Northrop C. Traumatic bronchial rupture. AJR Am J Roentgenol. 1980;134(6):1189-1193.

16. Hamad AM, Elmistekawy E, Elatafy E. Chronic atelectasis of the left lower lobe: a clinicopathological condition equivalent to middle lobe syndrome. Interact Cardiovasc Thorac Surg. 2012;15(4):618-621.

17. Sehitogullari A, Sayir F, Cobanoglu U, et al. Surgical treatment of right middle lobe syndrome in children. Ann Thorac Med. 2012;7(1):8-11. 